11

الإطار النظري لتقييم المشروعات الزراعية مع التركيز علي مشروعات المزارع السمكية ودور ها في تحقيق الأمن الغذائي

\author{
إيمان السيد محمد عبد الفتاح \\ معهد بحوث الاقتصاد الزراعي - وحدة بحوث الاقتصاد الزراعي- القاهرة .
}

يعتبر الاقتصـاد الزر اعي المصدر الرئيسي لإمداد السكان باحتياجاتهم من السـلع الغذائية.

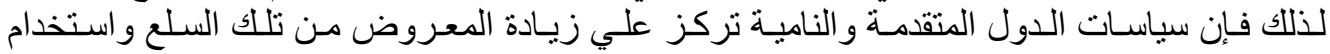

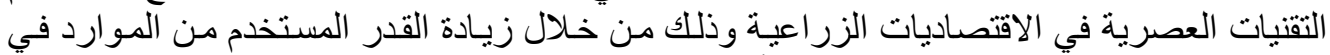

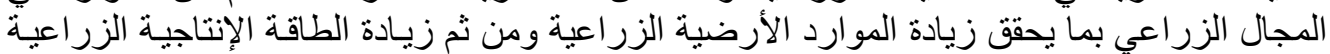

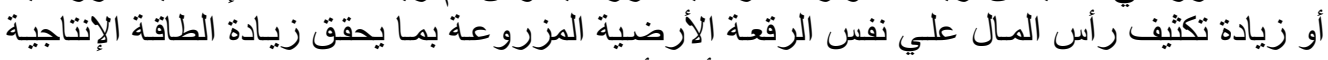

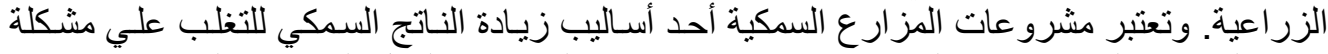

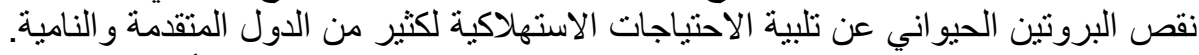

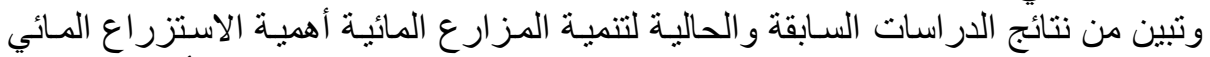

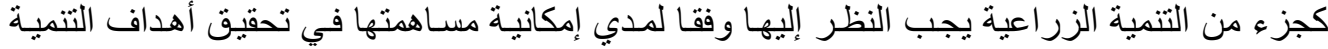

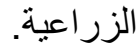

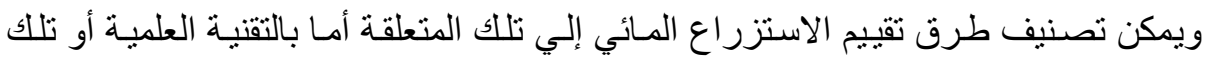

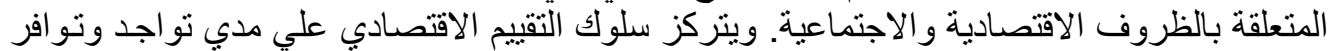

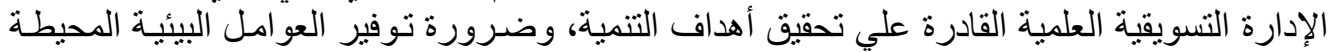

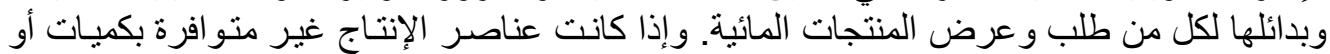

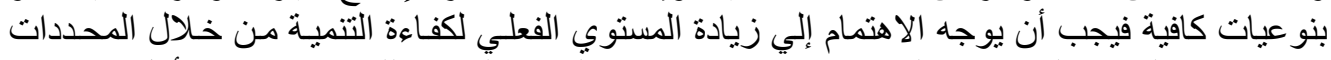

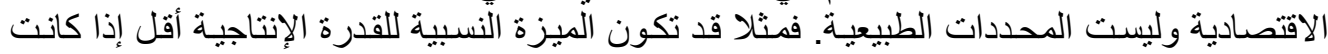

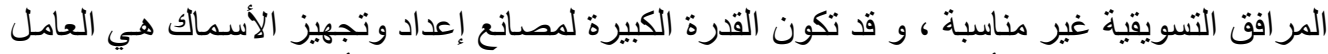

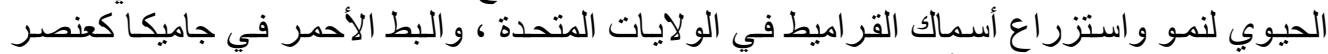

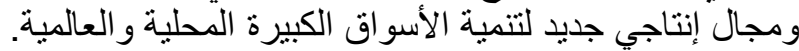

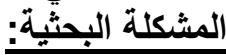

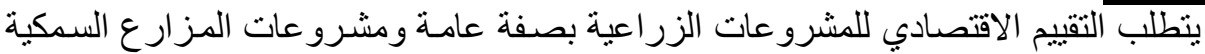

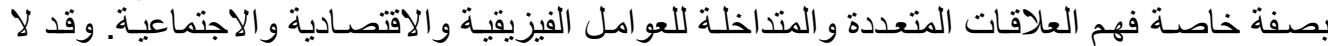

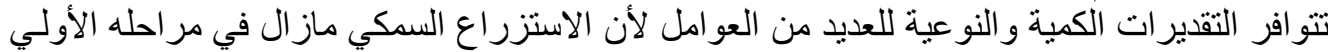

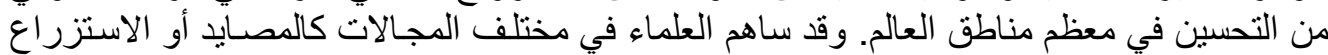

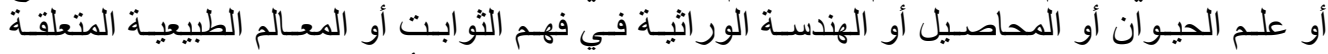

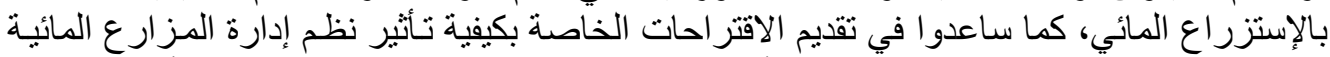

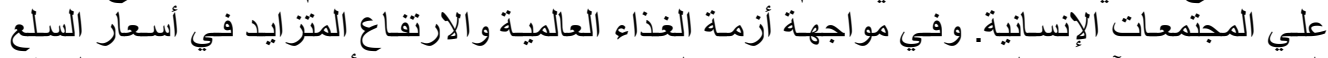

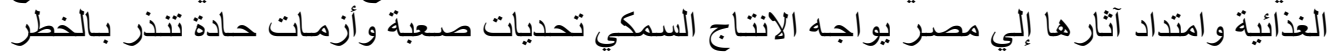

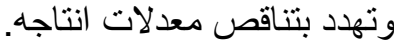

الهزف من البحث:

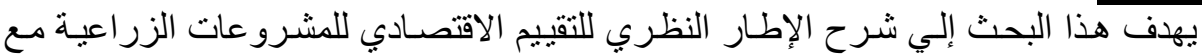

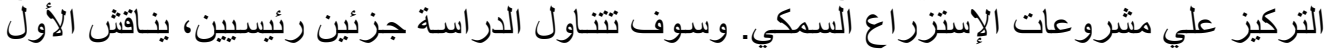

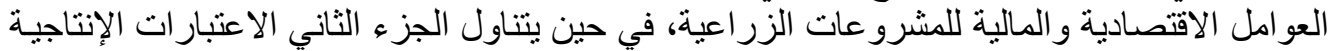

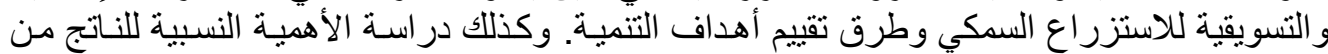

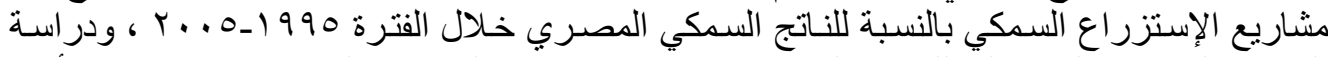

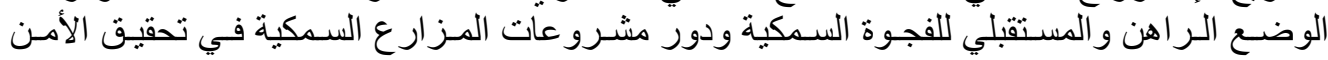

Fayoum J. Agric. Res. \& Dev., Vol.23, No.1, B. January, 2009 


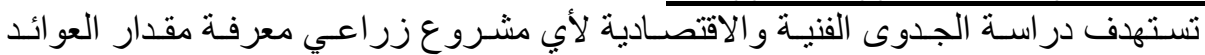

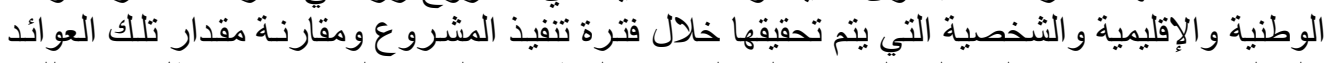

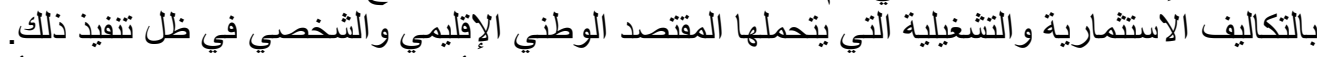

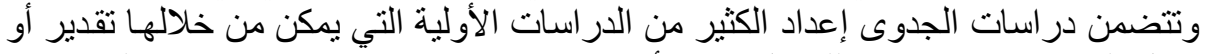

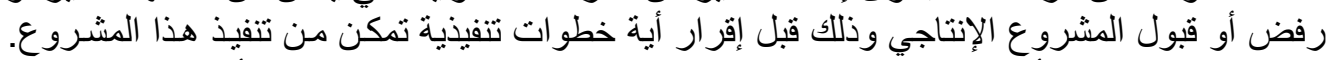

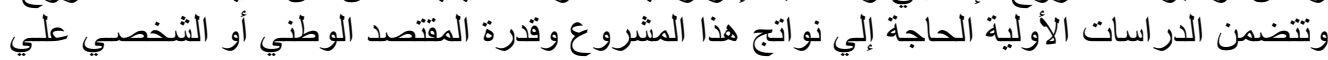

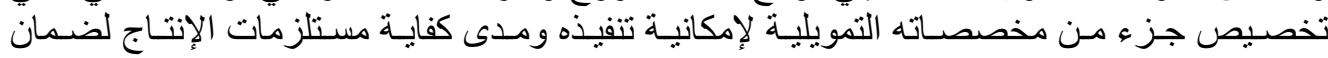

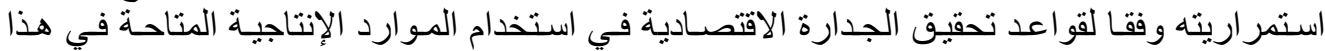

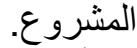

ثم تأتي مرحلة الجدوى النهائية للمشروع الذي أقرت جدواه الأولية من خلال إجر اء دراسـة تفصيلية

- مراسة وتقبر الطلب علي منتجات هذا المشروع

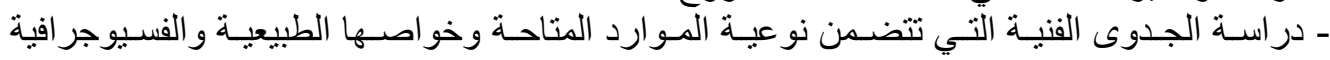

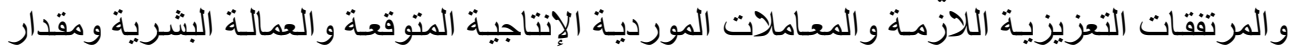
الاستثمار ات الر أسمالية والتشغيلية خلال فترة الإلية العمر الإنتاجي للمشروع.

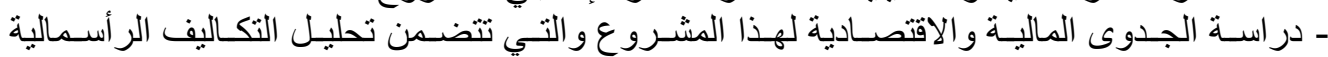

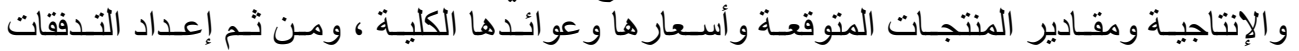

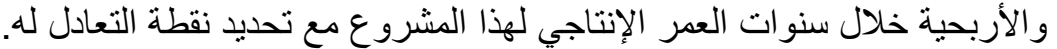

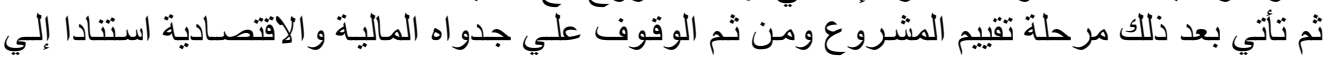

ه.

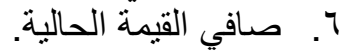

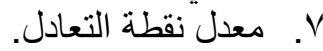

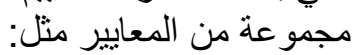

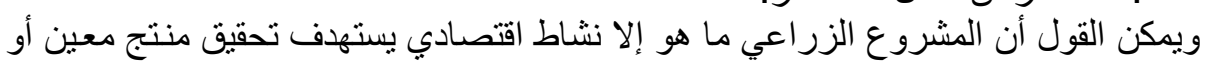

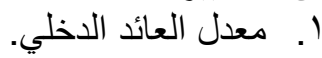

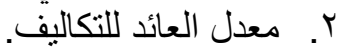

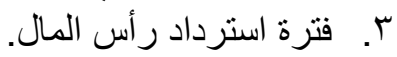

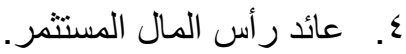

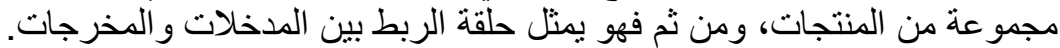

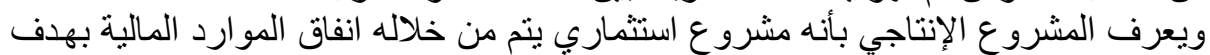

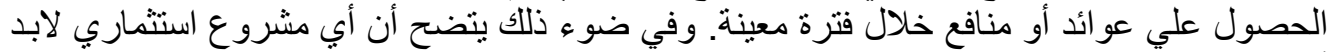

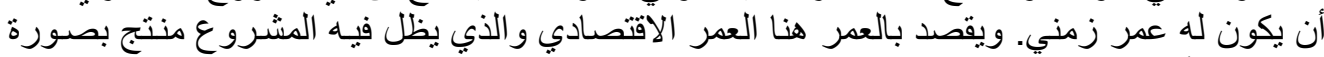

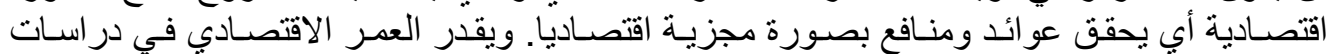

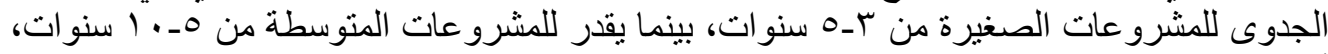

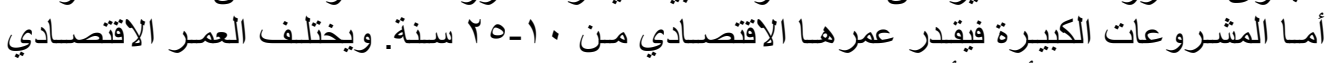

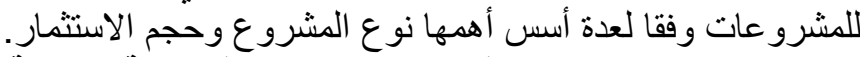

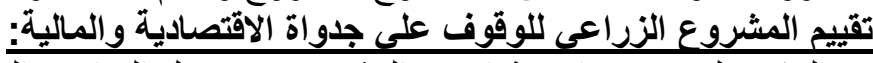

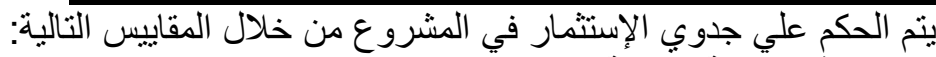

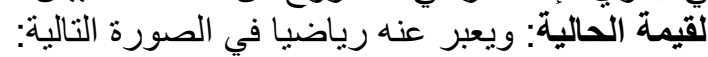

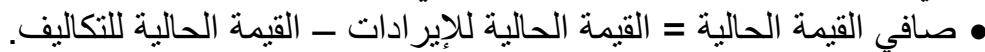

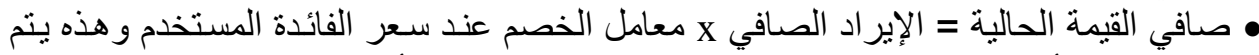

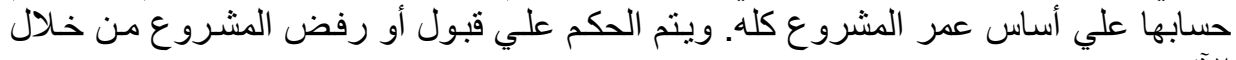
- الآتي: كان صافي القيمة الحالية موجب أي أن القيمة الحالية للإيرادات أكبر من القيمة الحالية للتكاليف يقبل المشروع ويتم تنفيذه.

Fayoum J. Agric. Res. \& Dev., Vol.23, No.1, B. January, 2009 
$r$.

- - ـ إذا كان صـافي القيمة الحالية سالب أي أن القيمة الحاليـة للإيـر ادات أقل مـن القيمـة الحالية

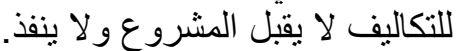

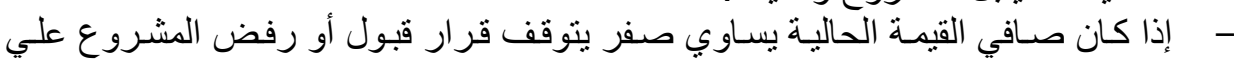

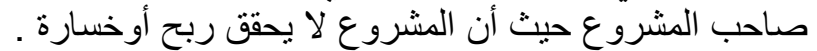

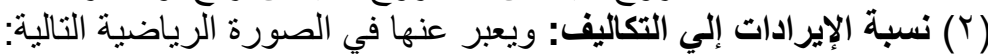

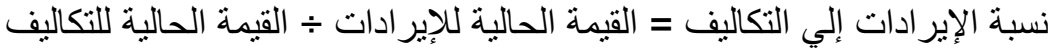

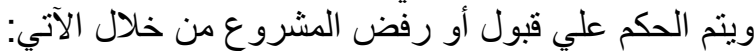

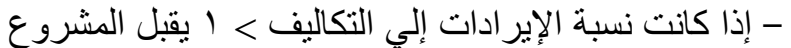

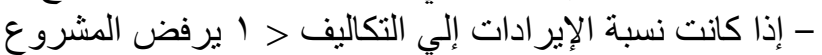

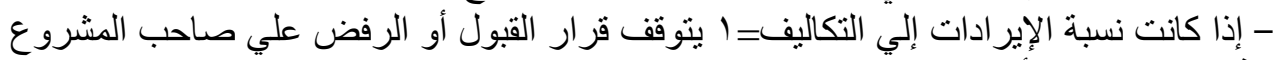

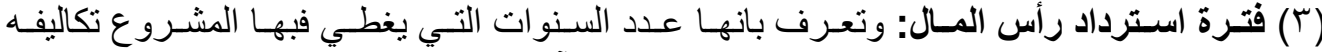

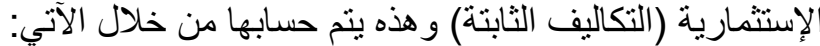

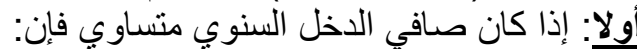

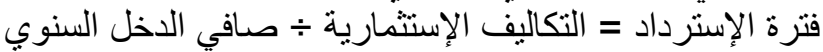
ثانيا: إذا كان صافي الدخل السنوي متغير فإن:

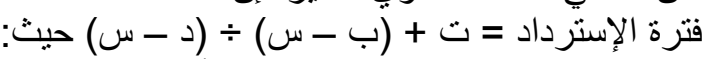

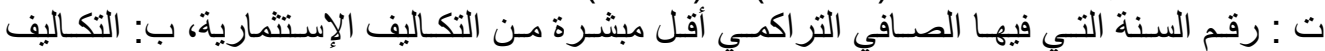
الإستثمارية

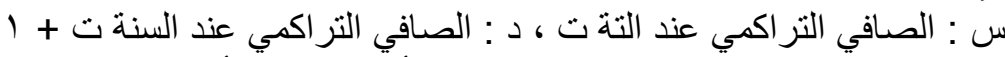

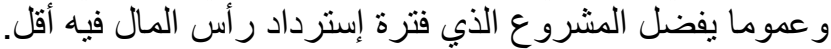

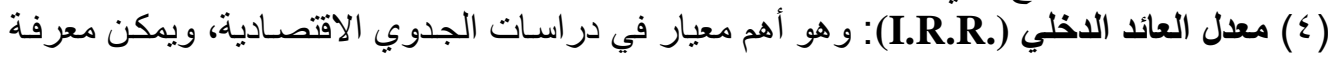

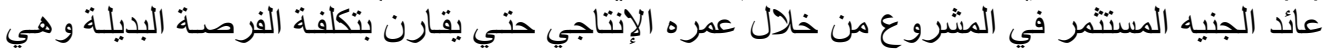

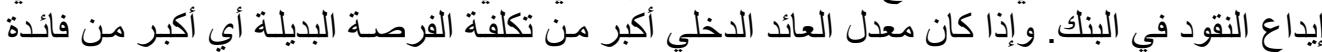
البنك يقبل المشروع و العكس صحيح. ويتم حسابه كالآتي:

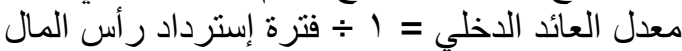

الاعتبار ات الإنتاجية والتسويقية لتنمية نظام ملائم للاستزراع العزية السمكي:

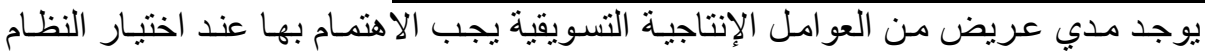

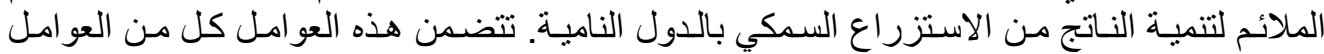

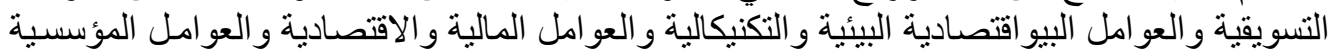

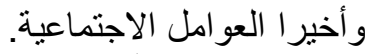
العوامل التسويقية: العوائية

يمكن تقسيم مشروعات الاستزراع المائي إلي عدد من المراحل الإنتاجية مثل التكاثر

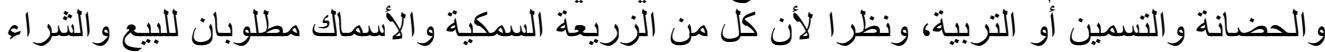

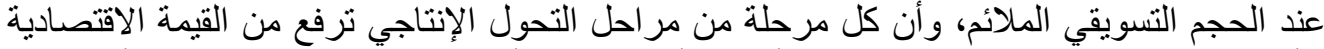

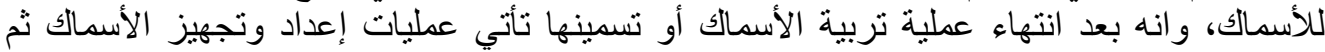

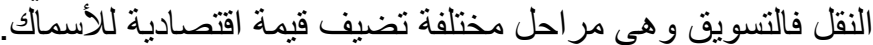

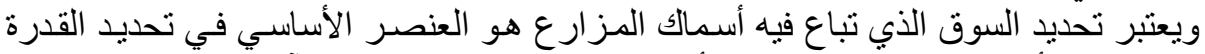

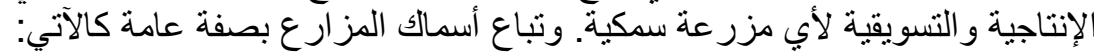

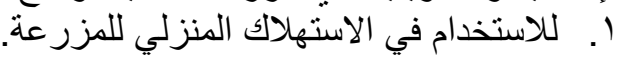
r

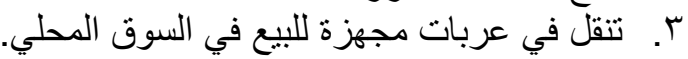
ع. ت. تباع للوسطاء. ه. تبـاع لمصـانع تجهيز الأسـماك لإعـدادها وتغليفهـا للبيـع النهـائي سـواء للاسـتهلاك المحلـي أو الخارجي.

Fayoum J. Agric. Res. \& Dev., Vol.23, No.1, B. January, 2009 
r)

و أن معرفة صفات الناتج وسلوك المستهلكين في السوق المختار هي أسس اختيـار أنسـب ونس

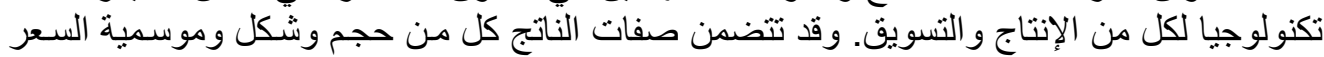

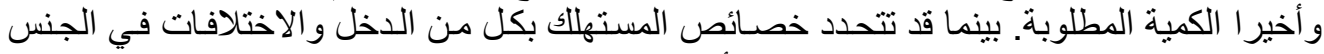

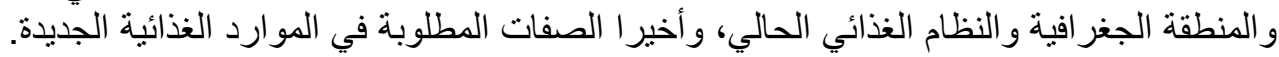

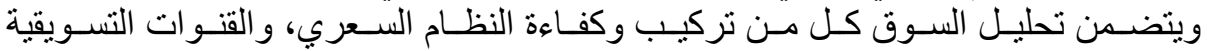

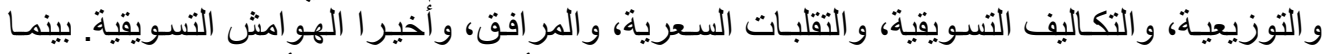

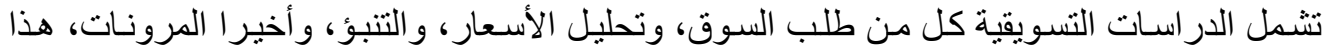

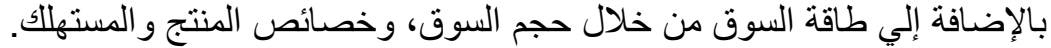

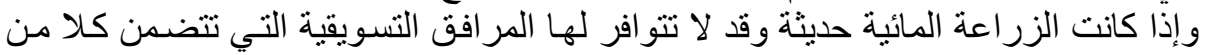

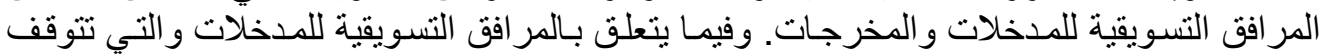

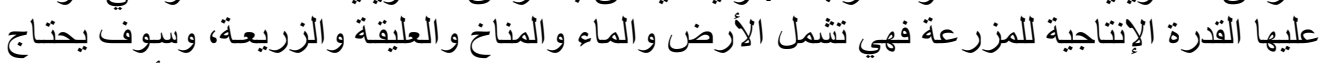

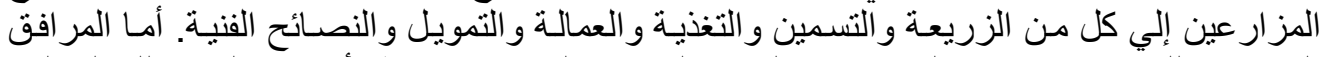

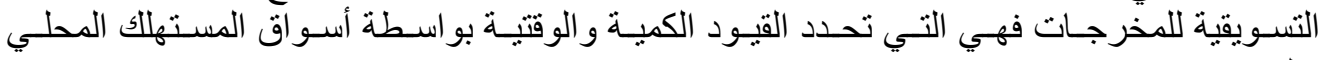
والخارجي.

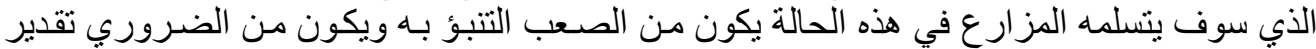

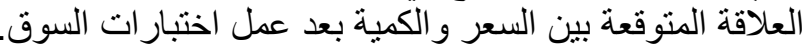

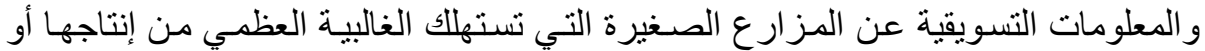

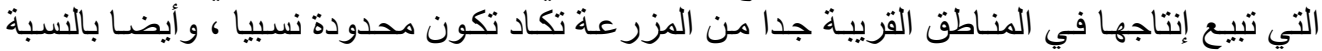

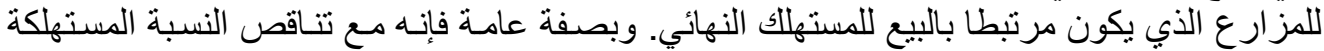

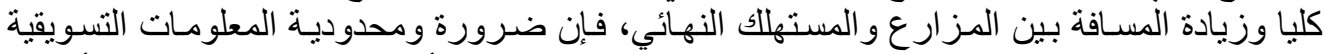

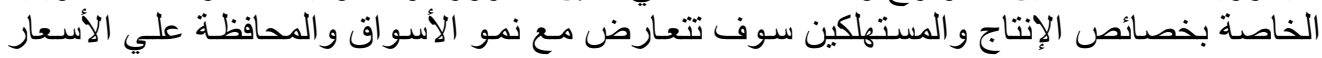
المرضية للمنتجين. العوامل الفنية والبيو اقتصادية: لمئة:

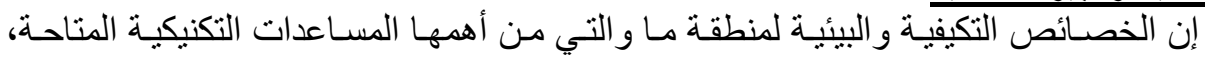

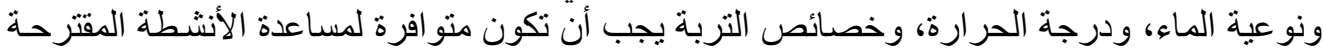

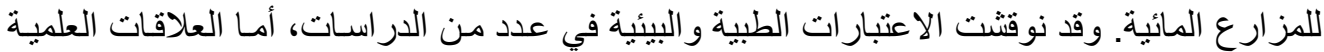

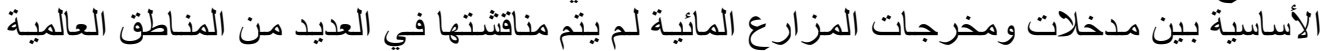

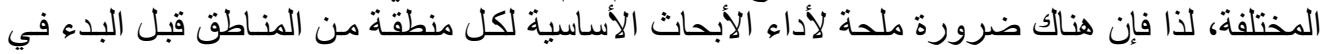

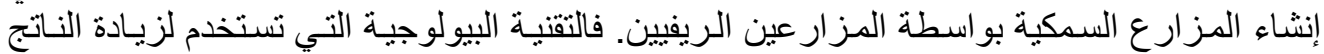

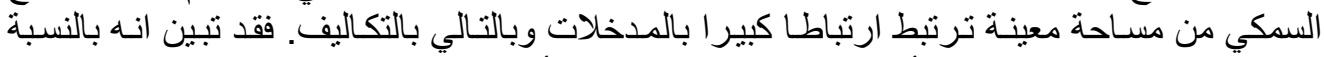

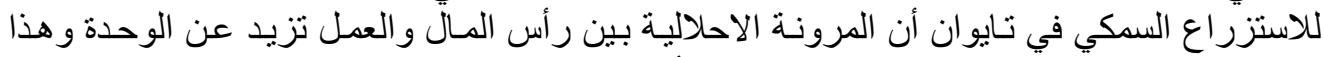

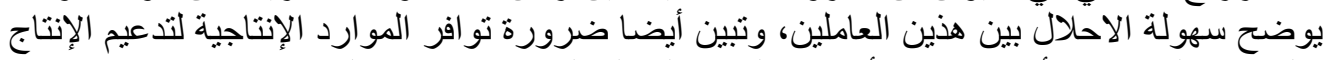

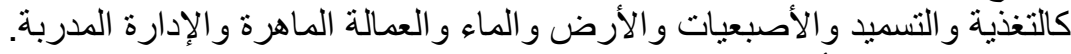

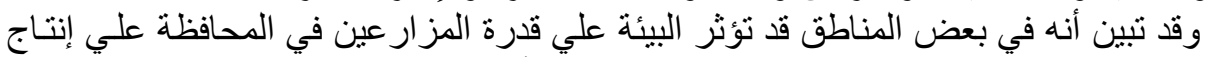

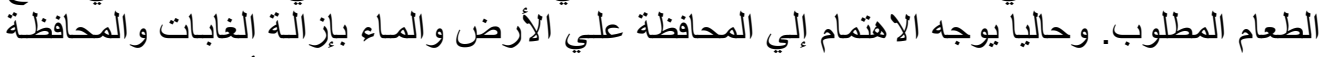

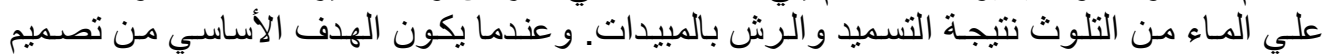

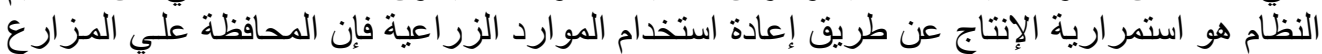

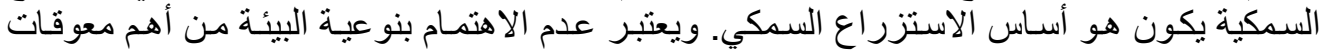

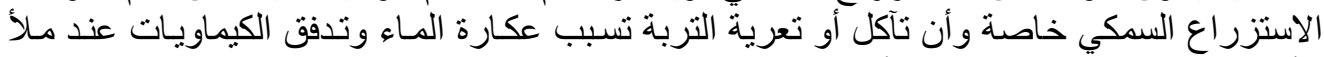

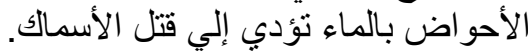

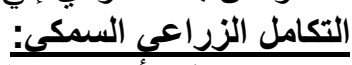

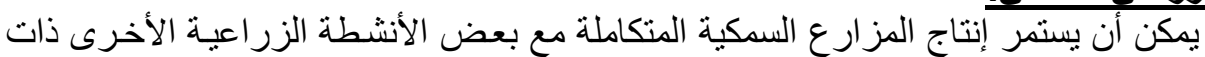

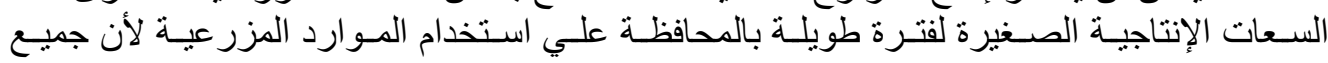

Fayoum J. Agric. Res. \& Dev., Vol.23, No.1, B. January, 2009 


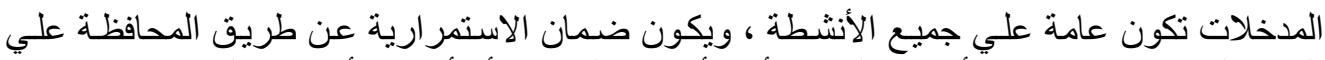

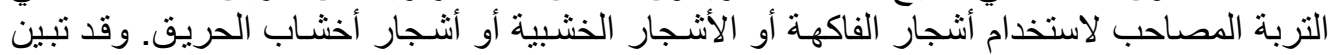

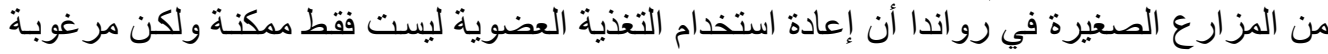

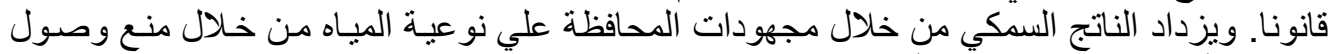

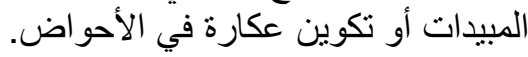

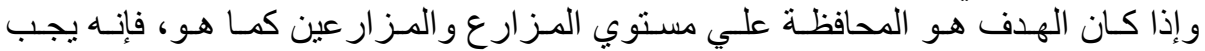

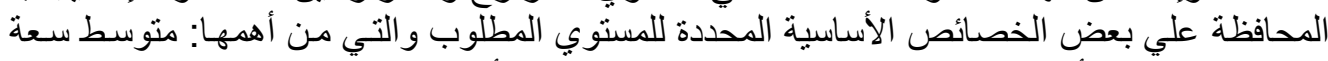

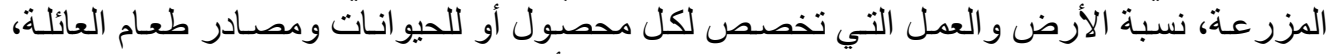

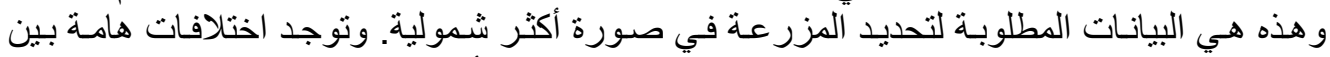

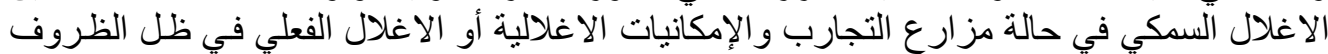

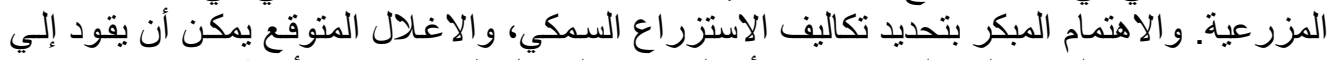

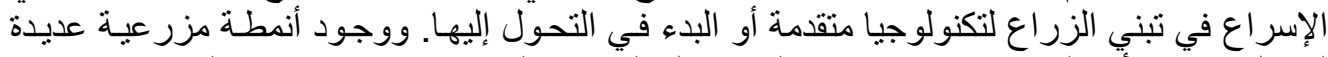

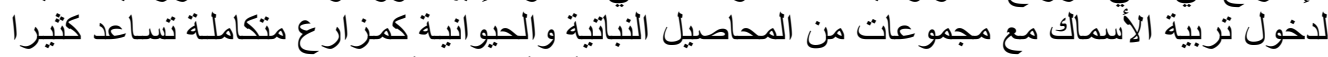

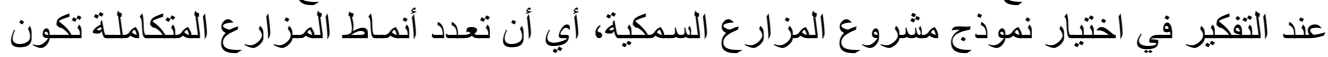

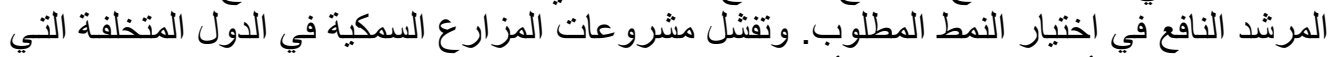

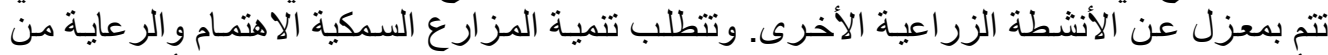

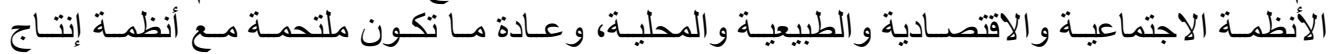

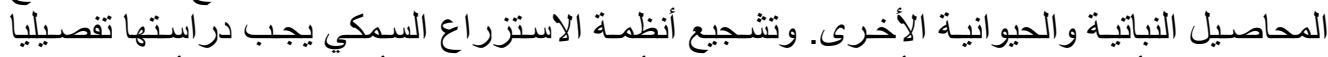

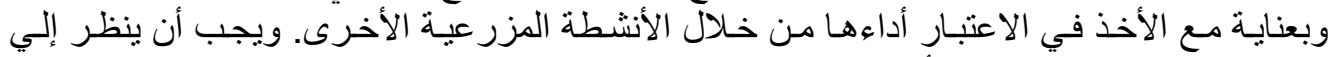

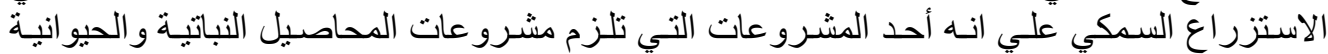

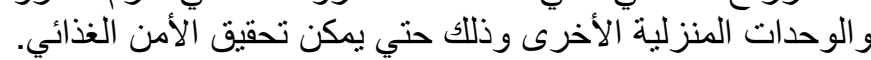

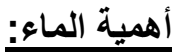

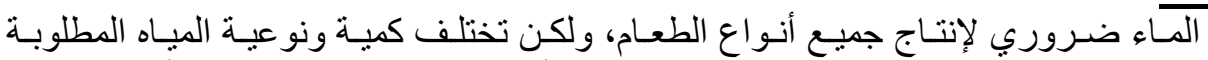

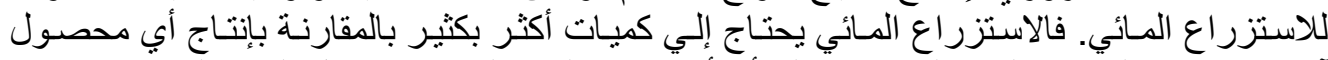

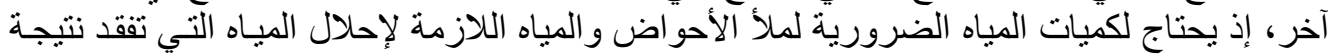

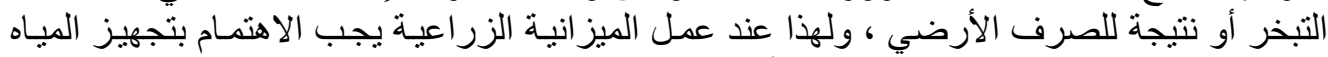
اللازمة للزر اعة المائية وري المحاصيل والأيخي وأخير ا التربية الحيو النية.

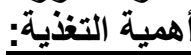

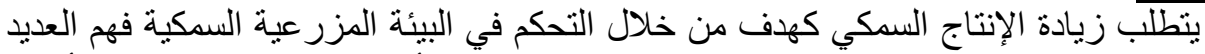

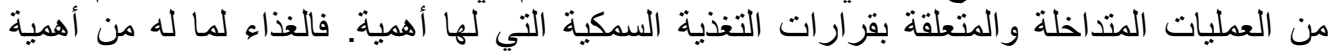

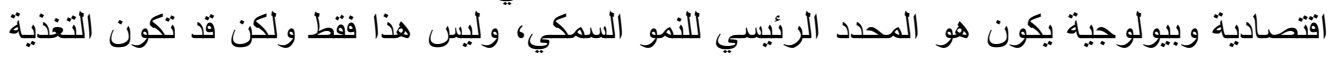

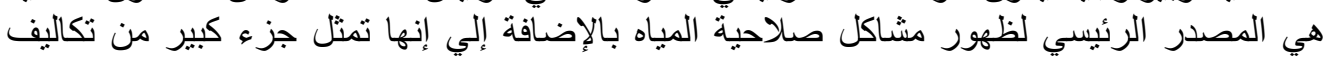

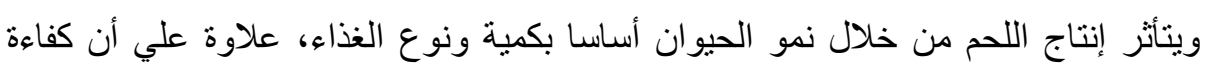
الإنتاج.

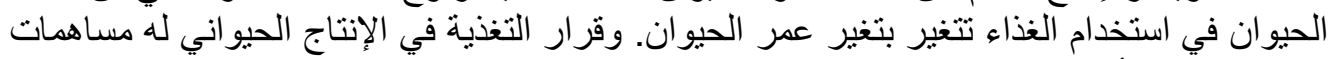

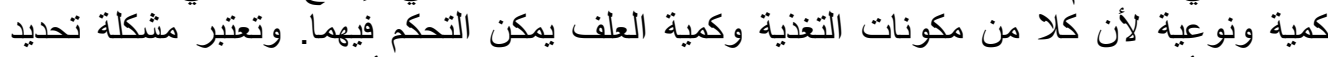
المقدار الأمثل من الغذاء وتوزيعه خلال عمر الحيوان والوقت من الون أهم المشاكل التي تم بحثها

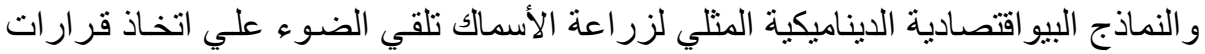

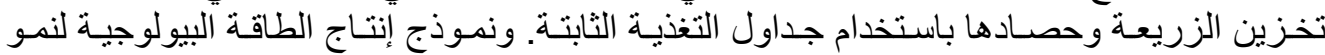

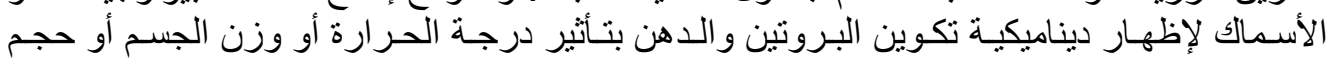

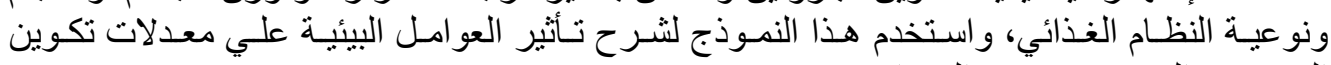
البروتين و الدهون في جسم الحيو وان.

Fayoum J. Agric. Res. \& Dev., Vol.23, No.1, B. January, 2009 
rr

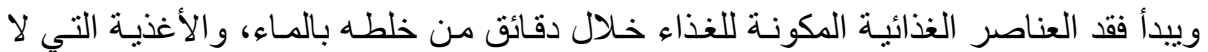

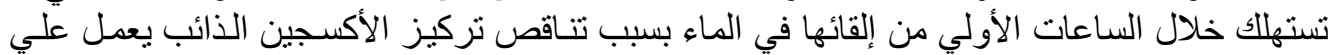

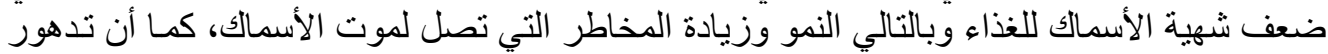

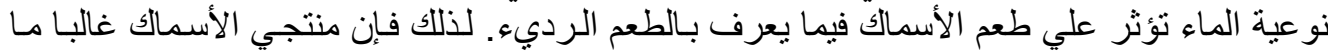

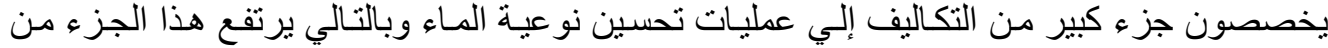

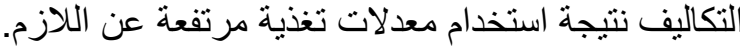

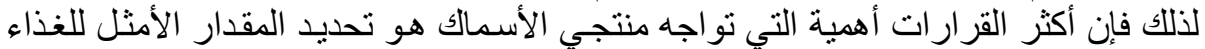

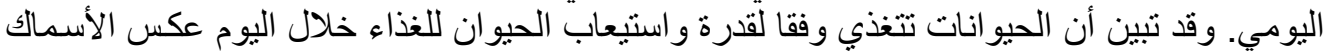

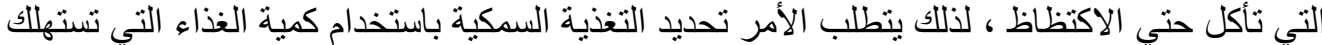

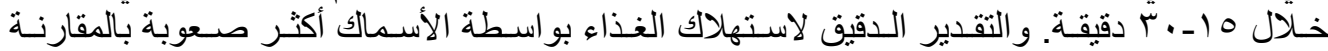

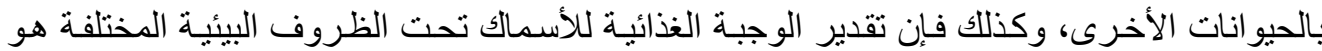

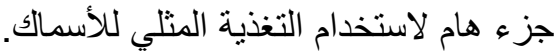

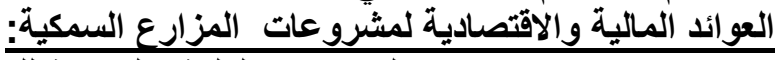

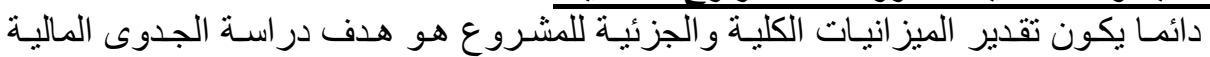

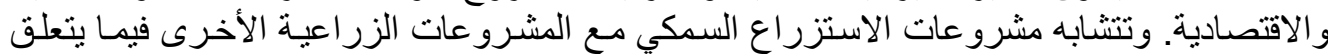

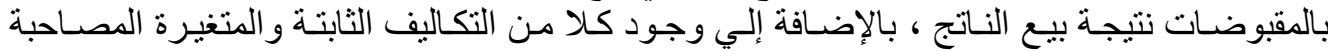

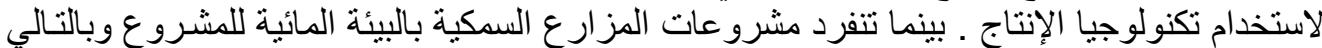
العو امل التكنولوجية والاقتصادية والانتاجية الاجتماعية.

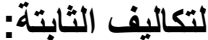

بنجاح تنمية الإنتاج و السوق يلزم توجيه استتمار ات معينة إلى كل من المدخلات و الخدمات

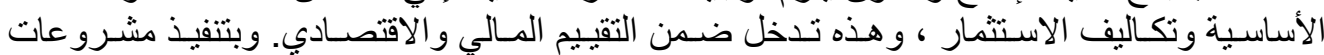

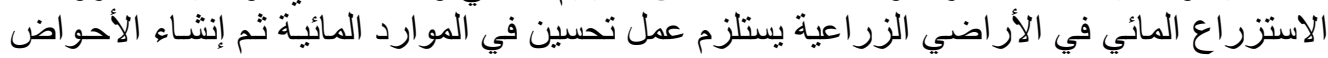
وتوفير بعض المعدات الخاصة بالاستزر الاع الاعن المائي.

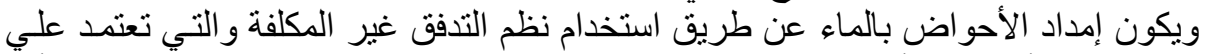

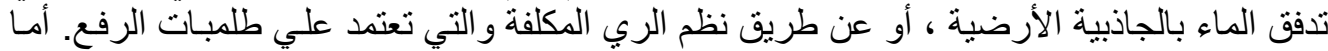

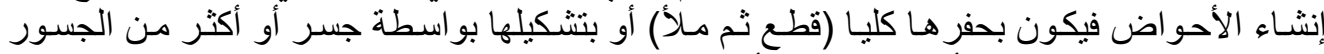

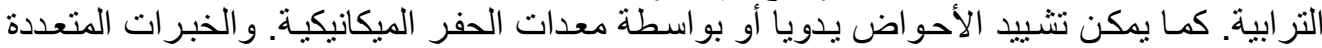

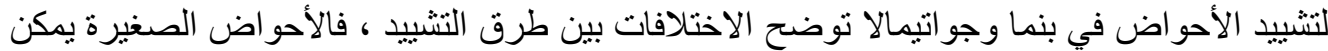

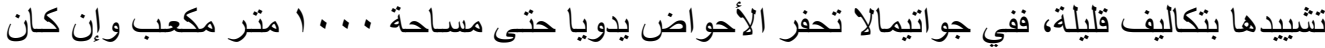

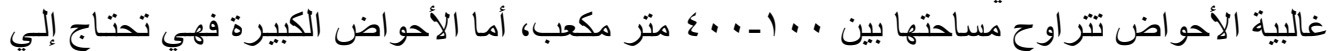

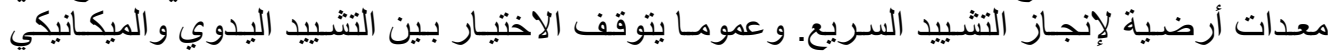

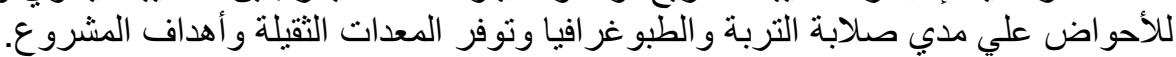

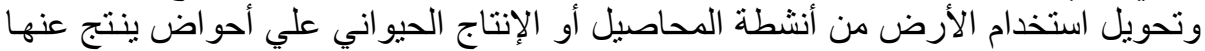

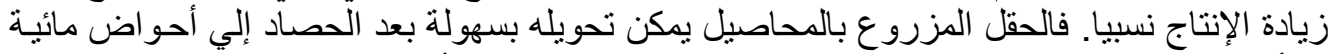

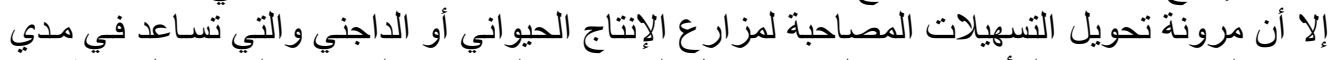

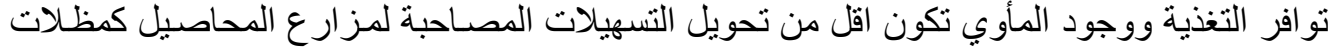

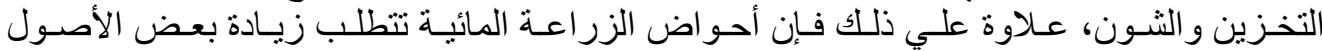

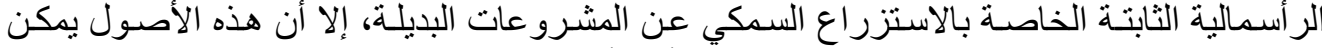

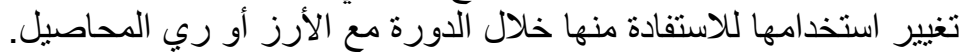

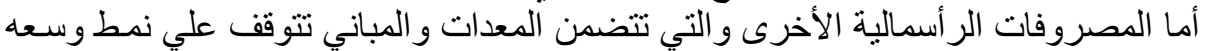

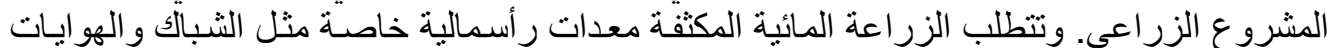

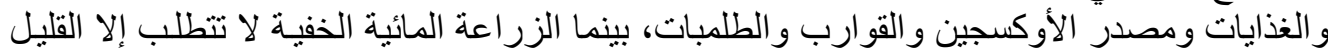

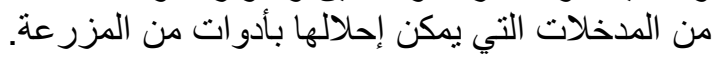

Fayoum J. Agric. Res. \& Dev., Vol.23, No.1, B. January, 2009 
Y $\varepsilon$

ويجب أن تؤخذ تكاليف الأرض في الاعتبار سواء في حالة شراء أو تأجير الأرض. كما

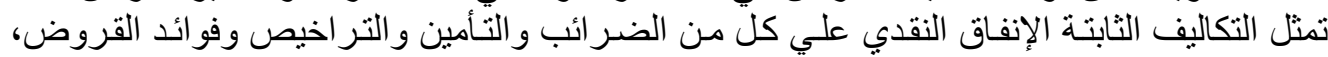

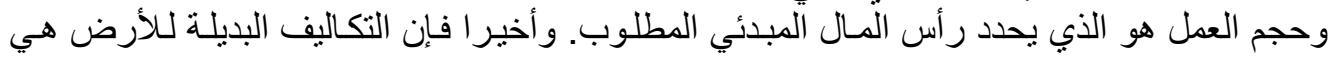

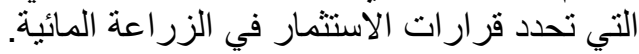

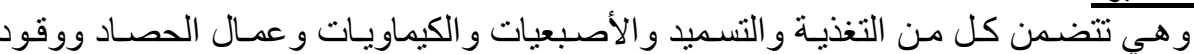

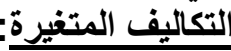

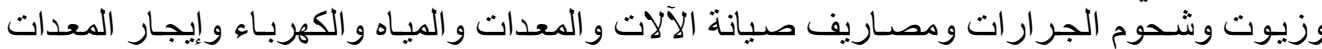

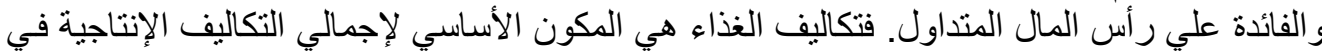

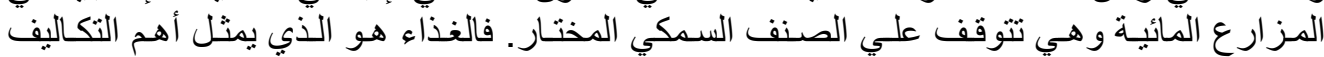

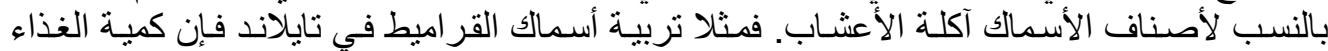

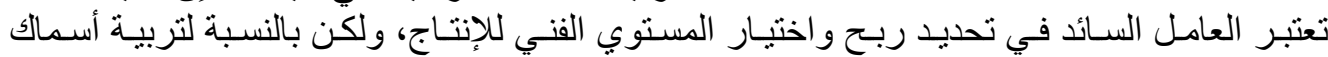

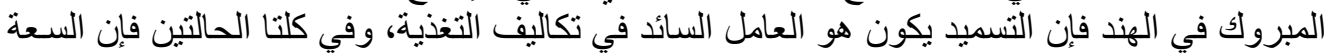

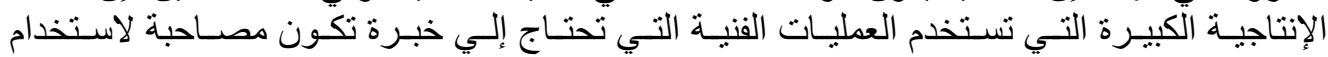
المستويات المرتفعة من التغذية.

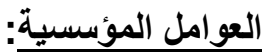

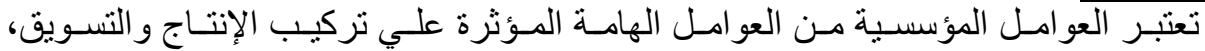

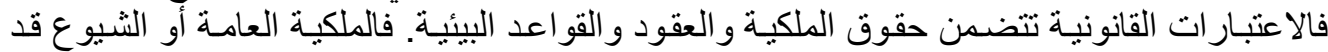

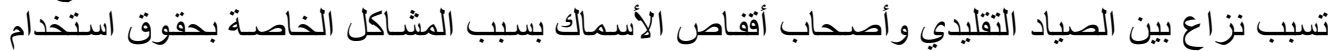

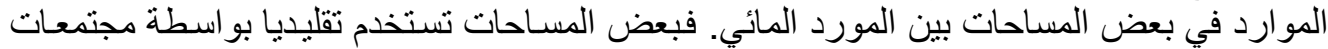

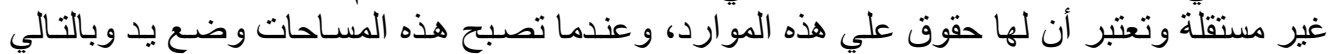

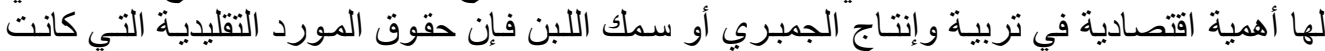

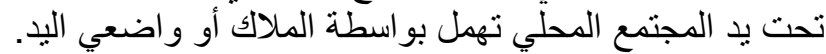

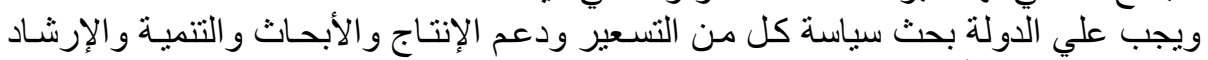

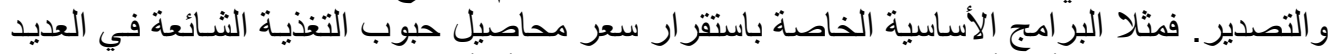

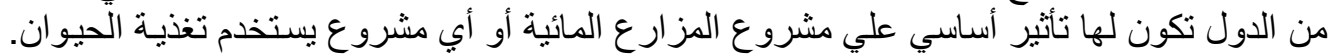

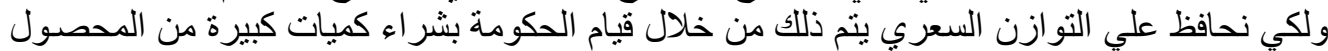

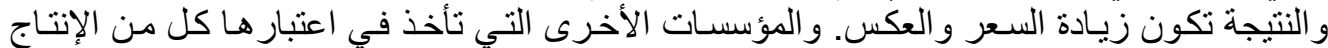

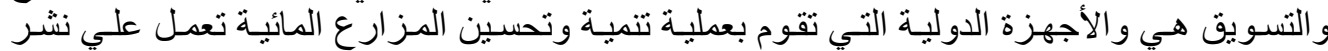

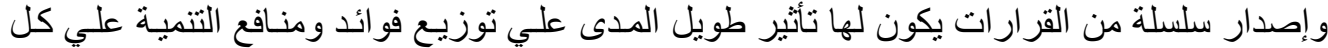

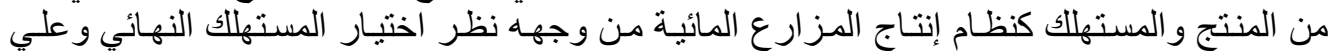
درجة و اتجاه تركيز رأس المال.

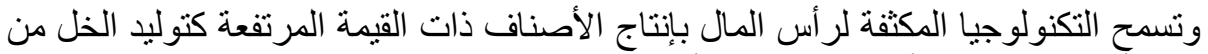

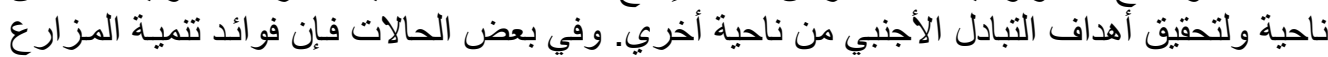

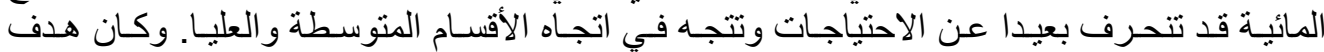

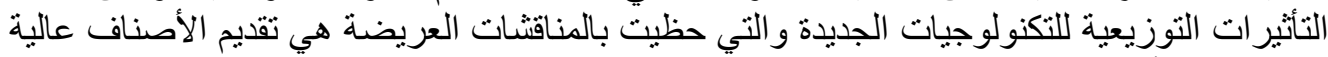
الإنتاج من الأرز و الذرة و القمح. الدراسة التطبيقة لمشاريع الإستزراع السمكى وأهميتها في تنمية مصادر الناتج السمكى المصري:

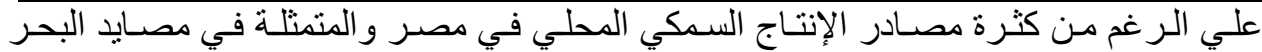

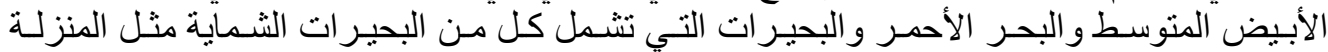

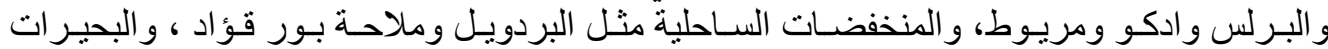

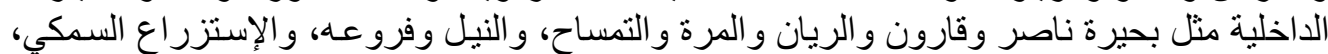

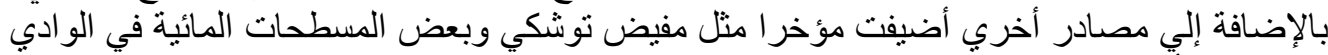

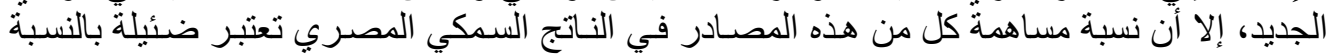

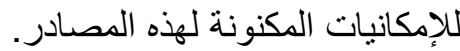

Fayoum J. Agric. Res. \& Dev., Vol.23, No.1, B. January, 2009 


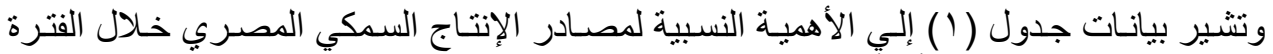

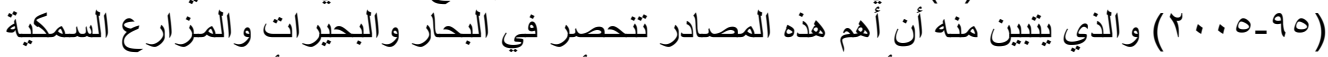

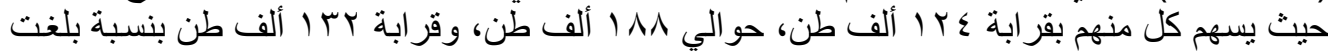

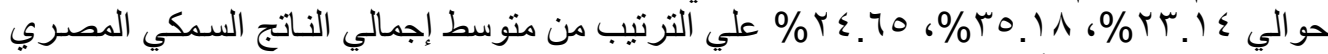

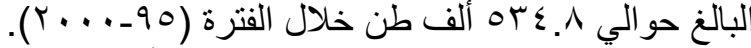

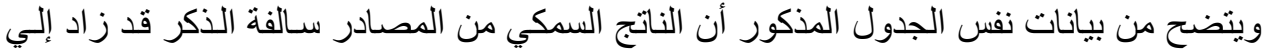

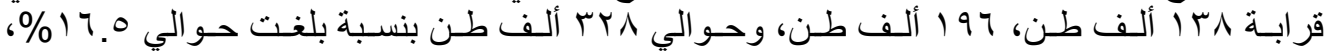

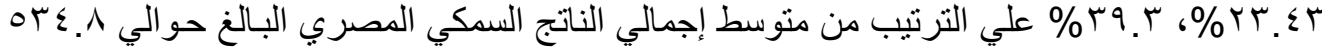

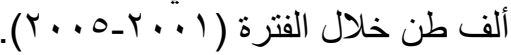

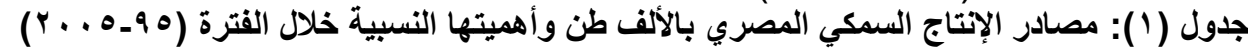

\begin{tabular}{|c|c|c|c|c|c|c|c|c|}
\hline \% للتغير & مقدار التخغير & $\%$ & $\begin{array}{l}-90 \\
r \ldots .\end{array}$ & $\%$ & $\begin{array}{r}-r \ldots l \\
r \ldots o\end{array}$ & $\%$ & $\begin{array}{l}-90 \\
r \ldots .\end{array}$ & المصدر \\
\hline $11 . r 1$ & $1 \leqslant .$. & 19.9 & $1 \% \cdot . v 0$ & 17.0. & ITV.Vo & $r T .1 \varepsilon$ & ITr.Vo & بحار \\
\hline r.q & $v . \varepsilon$. & $r \Lambda_{.} \cdot r$ & 191.10 & $r$ r. & 190.00 & ro.11 & 111.10 & بحيرات \\
\hline 10.11 & rr.A. & 10.04 & 1.7 .10 & 17.00 & $1 \pi \Lambda .10$ & $1 \% .9$. & $V \leqslant . r_{0}$ & مياه عذبة \\
\hline $1 \leqslant \Lambda . V \Gamma$ & 197.1. & rT.Os & rrq.q. & rq.r. & TrV.90 & $r \leq .70$ & $1 \pi 1.10$ & مزارع سمكية \\
\hline 111.17 & 11.74 & r.Ar & 47.7 & $\varepsilon . Y \leqslant$ & ro.rv & r.1r & 17.20 & حقول أرز \\
\hline $07 . \cdot \varepsilon$ & KYq.V. & 1.. & $7 \wedge \leq . \wedge T$ & $1 \ldots$ & Ar & 1... & $0 T \leqslant . \wedge V$ & الإجمالي \\
\hline
\end{tabular}

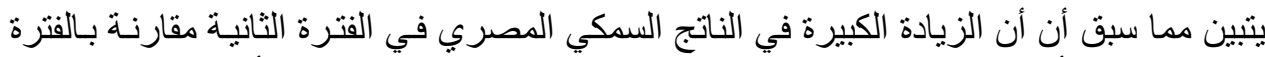

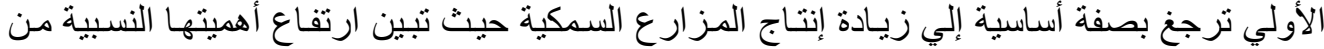

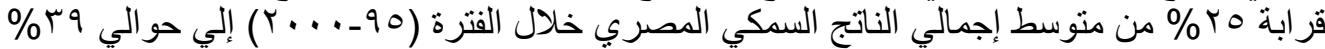

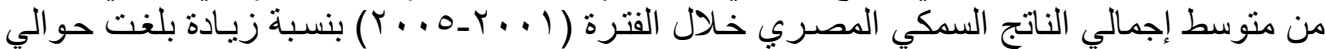

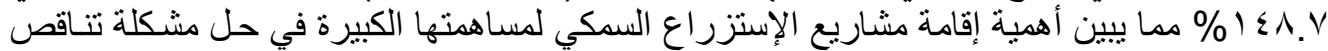

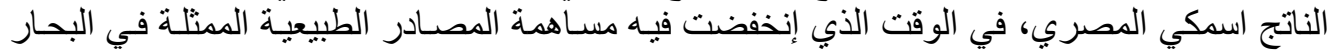

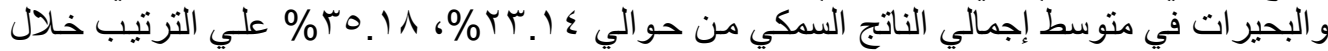

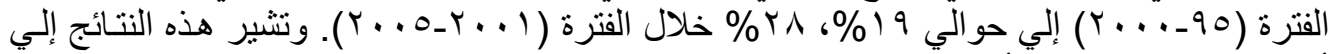

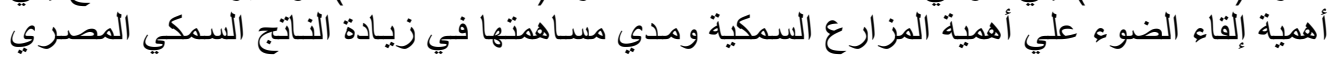

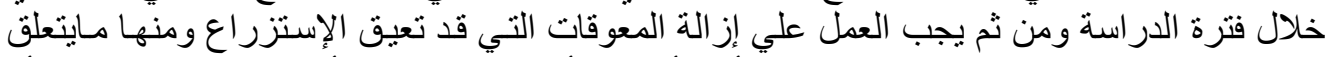

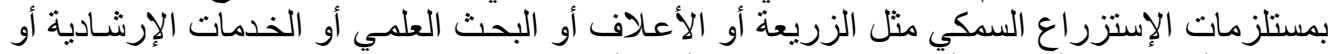

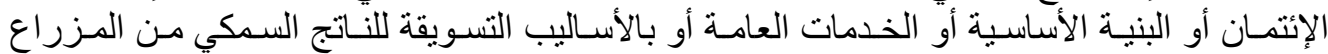

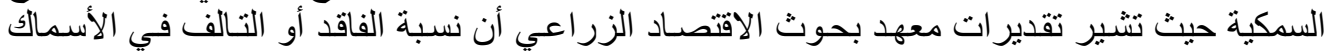

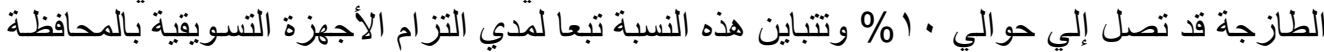

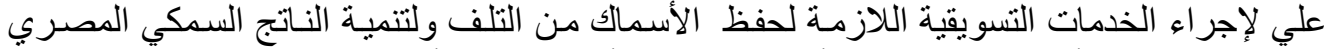

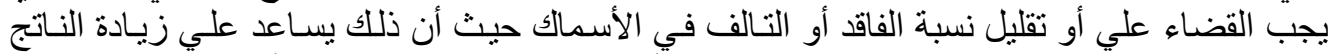

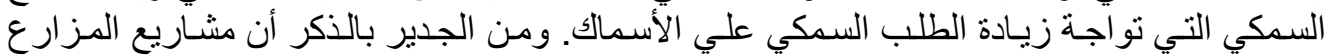

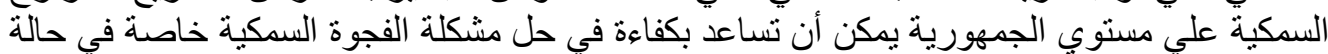

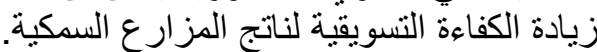

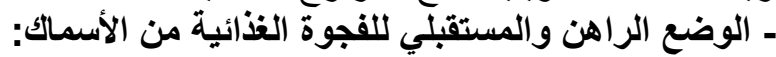

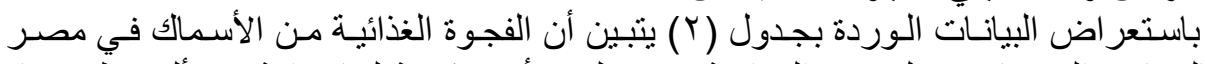

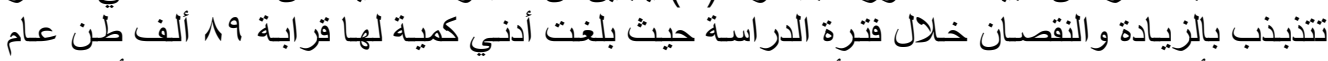

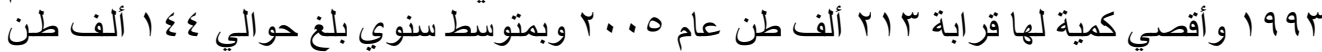

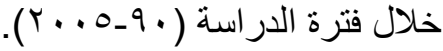

Fayoum J. Agric. Res. \& Dev., Vol.23, No.1, B. January, 2009 
ry

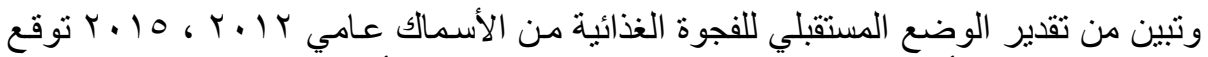

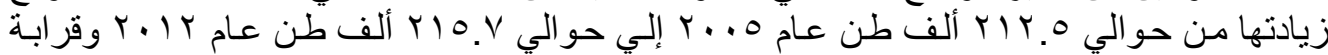

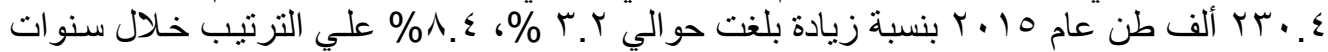

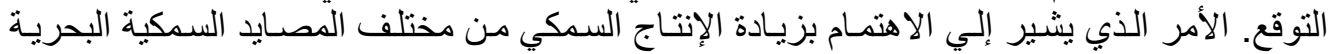

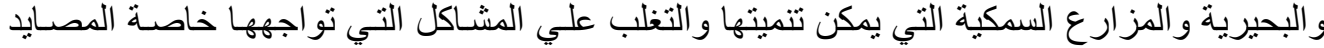

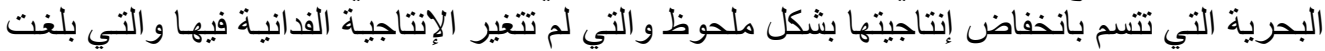

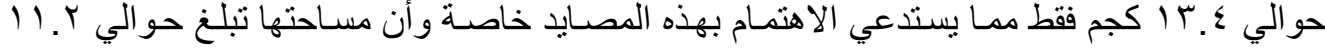

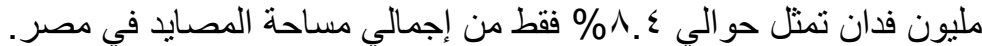

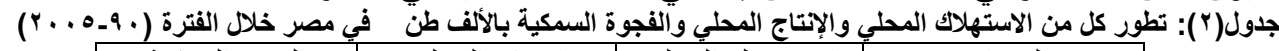

\begin{tabular}{|c|c|c|c|}
\hline الفجوة السمكية & الإنتاج المحلي & الاستهلاك المحلي & السنوات \\
\hline $1 \pi \leq .7$ & r9o.r & $\varepsilon Y ৭ . \wedge$ & 199. \\
\hline $1 \leq .0$ & r90.0 & $\leqslant$ $\leqslant$. & 1991 \\
\hline 1.1 .0 & үаץ. & rqะ.^ & 1995 \\
\hline $1 \wedge .0$ & rr. & $\leqslant 10$. & 1994 \\
\hline $1 \leq 7 . V$ & r & $\leqslant$ ะง.。 & $199 \leq$ \\
\hline 174.9 & $\left.\varepsilon \cdot v_{.}\right)$ & ov1. & 1990 \\
\hline 91.5 & $\leq \Psi .1$ & orr. & 1997 \\
\hline 1.0. & $\varepsilon 0 V_{.}$. & OTY. & 1998 \\
\hline & $0 \leqslant 0.7$ & TVA. & 1991 \\
\hline $\mid \wedge v .1$ & $7 \leqslant \Lambda .9$ & Art. & 1999 \\
\hline r.T & $V Y \leq . \varepsilon$ & $100 . \mathrm{V}$ & $r \ldots$ \\
\hline$|V|$. & $V Y 1.0$ & $q \leq r .0$ & $r \ldots l$ \\
\hline 178.1 & 1.1 .0 & $979 . r$ & $r \ldots r$ \\
\hline 104.4 & NVo.0 & I. YA.V & $r \ldots r$ \\
\hline $1 \wedge \leq . V$ & 170.r & $1.0 .$. & $r \ldots \leq$ \\
\hline r) T.O & $17 \cdot .0$ & 1.VT.. & $r \ldots o$ \\
\hline $1 \leq \leqslant . \wedge$ & $00 \wedge . \vee$ & $V \cdot \varepsilon .0$ & المتوسط السنوي \\
\hline
\end{tabular}

المصدر: جمعت وحسبت من الجهاز المركزي للتعبئة العامـة والإحصـاء ـ نشرة إحصـاءات الإنتاج السمكي - القاهرة -

أعداد متفرقة.

قياس الكفاءة التسويقية لبعض أصناف الأسماك الناتجة من المزارع السمكية:

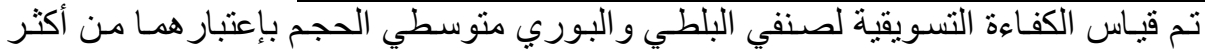

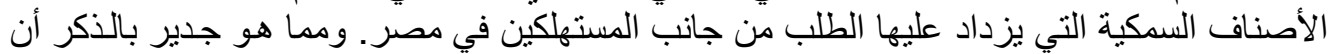

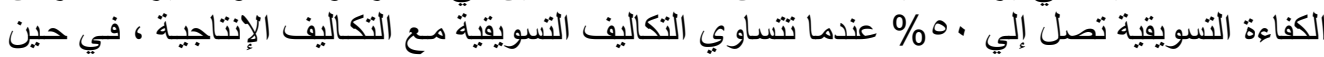

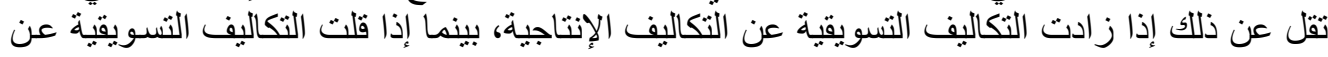

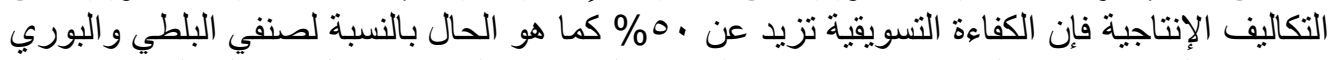
متوسطي الحجم موضع الدراسة. وتعتمد هذه الزيادة علي مقدار الفرق بين كل من التكـاليف الإنتاجيـة و التكاليف التسويقية.

وتثير نتائج تقدير الكفاءة التسويقية لكل من صنفي البلطي و البوري منوسطي الحجم الناتج

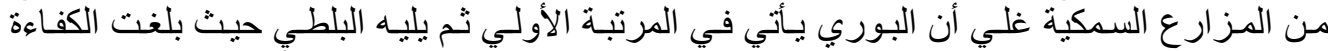

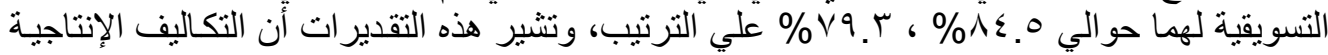

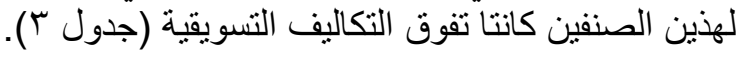

جدول (ب) : تقدير الكفاعة التسويقية لصنفي البلطي والبوري الناتج من المزارع السمكية

\begin{tabular}{|c|c|c|c|c|}
\hline 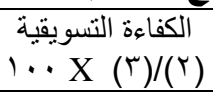 & الإجمالي & قرش/اليف الإنتاجية & المطلقة قرش/كجم ( التسويقية الكلية & البيان \\
\hline$\vee q . r$ & $T \vee A$ & orर & $1 \leq$ & البلطي \\
\hline$\Lambda \leqslant .0$ & 770 & סTr & $1 . r$ & البوري \\
\hline
\end{tabular}

( ) تثثير بيانات التكاليف الإنتاجية إلي متوسط تكلفة إنتاج كيلو جر ام سمك لمزرعتي برسيق و العباسة.

Fayoum J. Agric. Res. \& Dev., Vol.23, No.1, B. January, 2009 
rV

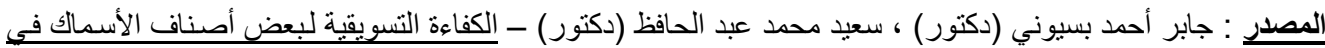

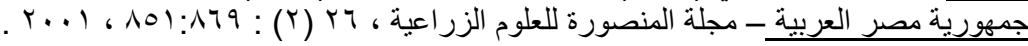

موجز الدراسة والتوصيات:

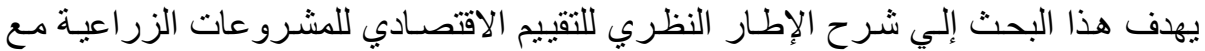

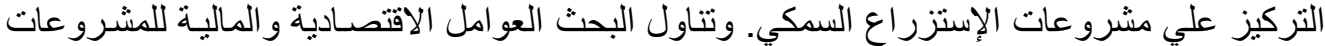

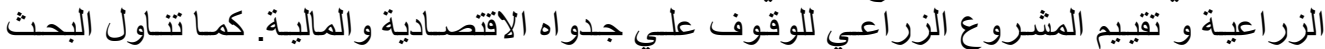

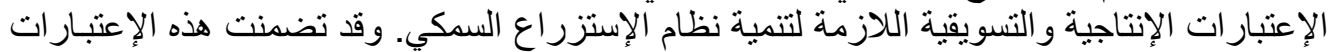

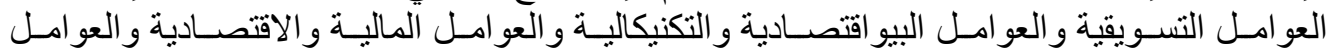
الإجتماعية

وقد تضمن البحث الدر اسة التطبيقية لمشـاريع الإستزر اع السمكي و أهميتها في تتمبـة مصسادر

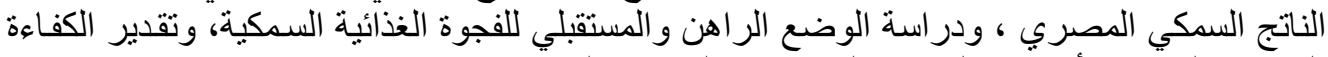

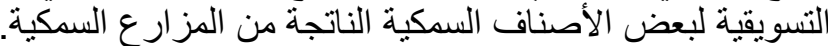

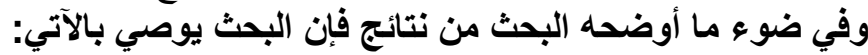

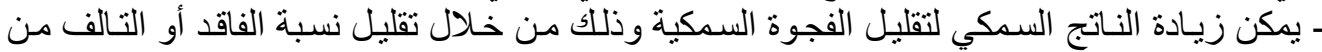
الأسماك خلال مر احله التسويقبة المختلفة ويتطلب ذلك تشجيع الإستثمار في الأساليب التسويقية مثل

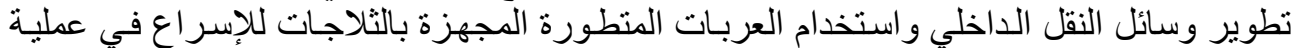

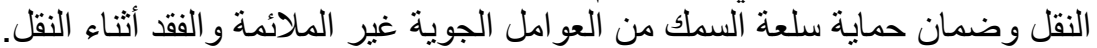

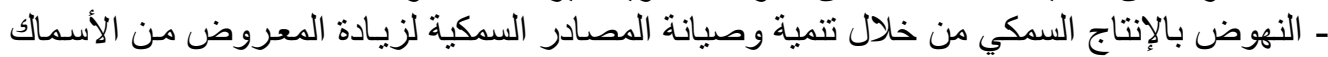
بإعتبار ها مصدر بروتيني هام لتوفير ها للمستهلكين وخاصة محدودي الإني الدخل بأسعار مناسبة ويمكن

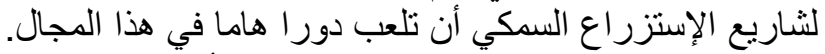

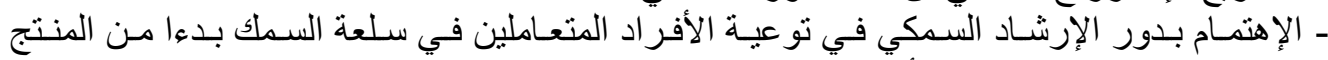
ووصو لا بالمستهلك النهائي بأهمية زيادة الناتج السمكي للتغلب علي مشكلة الفجوة الألئ السمكية.

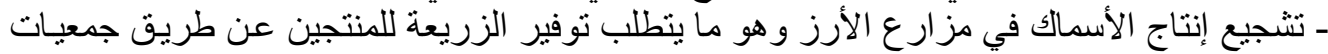
تعاونية متخصصة - رفع كفاءة المزارع السمكية (الأقفاص) وتنميتها وتشجيعها، شريطة عدم التوسع إلا في حدود المتاح

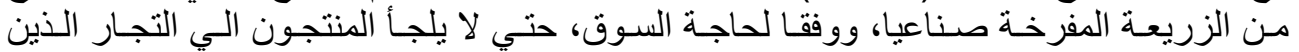

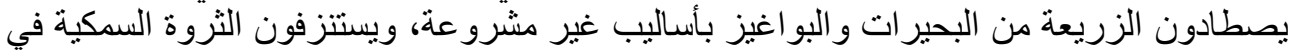
البحير ات، مع تشديد الرقابة بتنفيذ قانون الصيرات البواغيد الذي يمنع صيد أسماك الزريعة.

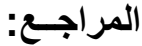

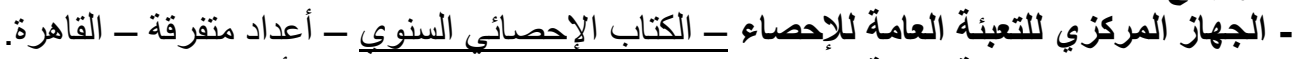

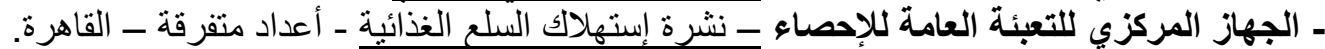

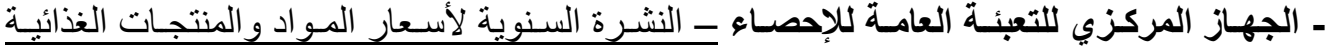

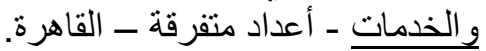
- جابر أحمد بسيوني (دكتور)، سـعيد محمد عبد الحسافظ (دكتور)، الكفاءة التسويقية لبعض أصذاف أصناف

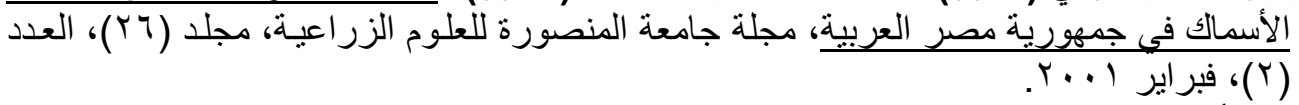

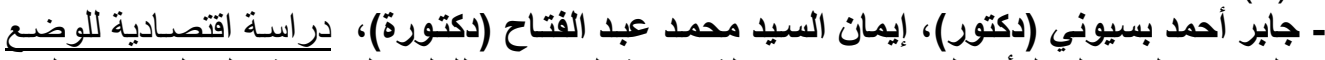

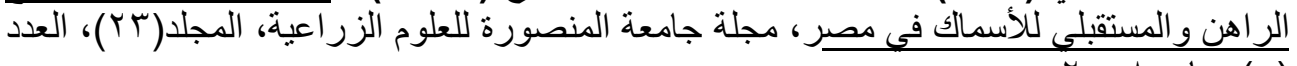

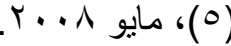

Fayoum J. Agric. Res. \& Dev., Vol.23, No.1, B. January, 2009 
r

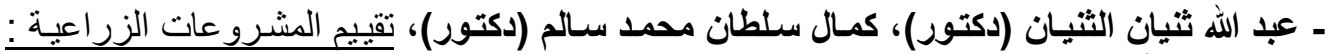

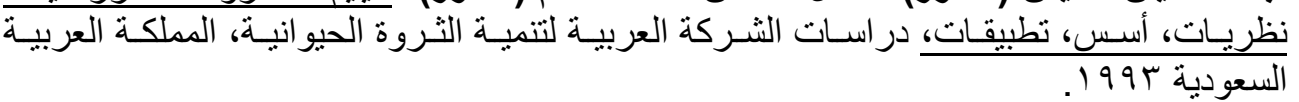

- Adel Y. Awad, Gaber A. Bassyouni ,Theoretical Framework of Economic Evaluation of Aquaculture Fish Projects, $7^{\text {th }}$ International Conference on Environment Protection is A Must, P.P. 5-26, 20-22 May, 1997, Alexandria, Egypt, Organized by National Oceanography and Fisheries, and International Scientists Association.

- Blaylock, F., and D. Smallwood (1986), U.S. Demand for Food: Household Expenditure, Demographics and Projection, Technical Bulletin No. 1713, Economic Research Service, U.S.D.A. Washington.

- Chen, H., and O. Capps, J. (1986), Demand Analysis of Fresh and Frozen Finfish and Shellfish in the United States, American Journal of Economics 70, 533-542.

- Engle, C., and U. Hatch, (1988), Economic Assessment of Alternative Aquaculture Aeration Strategies, Journal of the World Aquaculture Society, 19, 85-96.

- Gittenger, J., (1982), Economic Analysis of Agricultural Projects, The Economic Development Institute of the World Bank, Baltimore: Johns Hopkins University Press.

- Meada, J. (1989), Aquaculture Management, New York Van Nostrand Reinhold.

- Smith, L., and S. Peterson, (1982), Aquaculture Development in LDC: Social Economics and Political Problems, Boulder, Colorado: Westview Press.

- Stade, R., Hazella, P., and Bell, C., (1982), Project Evaluation in Regional Perspective: A study of An Irrigation Project in Northwest Malaysia, World Bank, The Johns Hopkins University Press, Baltimore.

\title{
THEORETICAL FRAMEWORK OF ECONOMIC EVALUATION OF AGRICULTURAL PROJECTS WITH EMPHASIS ON AQUACULTURE PROJECTS AND ITS ROLE TO ACHIEVE FOOD SECURITY
}

\author{
Iman E. M. Abd Elftah \\ Institute of Agric. Eco. Research - Cairo
}

\begin{abstract}
This research aims to investigate the theoretical framework of economic evaluation agricultural projects with emphasis on aquaculture
\end{abstract}

Fayoum J. Agric. Res. \& Dev., Vol.23, No.1, B. January, 2009 
projects. The study showed the importance of agricultural project, and agricultural project cycle which can be divided to four stages: pre-investment, investment, operation stage, and evaluation stage.

Assessment of the economic viability of aquaculture project requires an interdisciplinary understanding of physical, social and economic relationship. Quantitative or qualitative estimates of many relevant variables are not readily available because aquaculture is in early stages of development in most areas of the world.

The study comprised the outline aquaculture production-marketing consideration which includes marketing, technical-environmentalbioeconomic, institutional, and social respects.

The study showed that the, fish gap increased with annual rate of growth with $1.5 \%$ during the period net ween (1995 and 2005).

The most important fish production resources in Egypt is the seas, lacks and fish farms with 124 thousand ton, 188 thousand ton and 132 thousand ton or about $23.14 \%, 35.18 \%$ and $24.65 \%$, respectively from the annual average total of Egyptian fish production during the period (95-2000), and it increased to about 138 thousand ton, 196 thousand ton and about 328 thousand ton or about $16.5 \%, 23.34 \%$, and about $39.3 \%$ respectively from the annual average total of Egyptian fish production during the period (20012005). 